\title{
Anomalous diffusion in a quenched-trap model on fractal lattices
}

\author{
Tomoshige Miyaguchi ${ }^{1, *}$ and Takuma Akimoto $^{2}$ \\ ${ }^{1}$ Department of Mathematics Education, Naruto University of Education, Tokushima 772-8502, Japan \\ ${ }^{2}$ Department of Mechanical Engineering, Keio University, Yokohama 223-8522, Japan
}

(Dated: January 11, 2021)

\begin{abstract}
Models with mixed origins of anomalous subdiffusion have been considered important for understanding transport in biological systems. Here, one such mixed model, the quenched trap model (QTM) on fractal lattices, is investigated. It is shown that both ensemble- and time-averaged mean square displacements (MSDs) show subdiffusion with different scaling exponents, i.e., this system shows weak ergodicity breaking. Moreover, time-averaged MSD exhibits aging and converges to a random variable following the modified Mittag-Leffler distribution. It is also shown that the QTM on a fractal lattice can not be reduced to the continuous-time random walks, if the spectral dimension of the fractal lattice is less than 2.

PACS numbers: 87.15.Vv 05.40.Fb 02.50.Ey
\end{abstract}

Anomalous diffusion has received much attention in recent years $[1,2]$, because it has been reported in many single-particle tracking experiments in biological systems [3] and molecular dynamics simulations [4]. In particular, much effort has been devoted to theoretical studies to elucidate what kind of anomalous diffusion is consistent with these experiments [3-5]. Among these studies, models with mixed origins of anomalous diffusion are found to agree well with some experimental data. For example, continuous time random walks (CTRWs) on fractal lattices well reproduce the diffusion of potassium channels on plasma membrane $[6,7]$, and a mixed model of fractional Brownian motion (FBM) and CTRW well explains the diffusion of insulin granules in cells [8] as well as molecular dynamics simulations of water molecules on the membrane surface [9]. However, these theoretical models are almost phenomenological, and their underlying mechanisms still remain to be elucidated.

As biological origins of these mechanisms, fractal structures are considered to be generated by molecular crowding $[6,10]$; the FBM is believed to be due to viscoelasticity of the cytoplasm [8]. On the other hand, energetic disorder due to transient traps to binding sites is considered to generate the CTRW dynamics $[6,8]$. In fact, such energetic disorder is a physical origin of CTRWs for the case of non-fractal lattices with the spacial dimension larger than $2[11,12]$. However, little is known about such reduction for the diffusion on fractal geometry. Here, we study random walks in random energy landscapequenched trap model (QTM) [11-14] — on fractal lattices, and show that the reduction to CTRWs is impossible for the system with the spectral dimension lower than 2 . The results in this Rapid Communication are a generalization of Ref. [14], which studied the QTM on hypercubic lattices.

We consider random walks on a fractal lattice with fractal dimension $d_{f}$ and spectral dimension $d_{s}$. The

\footnotetext{
* tmiyaguchi@naruto-u.ac.jp
}

fractal dimension $d_{f}$ characterizes a static property (the configuration of the lattice points), while the spectral dimension $d_{s}$, originally defined through the spectral density of state, characterizes a dynamic property. Even the discrete time random walks (DTRWs) on fractal lattices show the anomalous diffusion [15]. We denote the position of the DTRW at time $n$ by $\tilde{\boldsymbol{r}}(n) \in \mathbb{R}^{d}$. Then, the ensemble-averaged mean square displacement (EMSD) of DTRWs on fractals is given by

$$
\left\langle\delta \tilde{\boldsymbol{r}}^{2}(n)\right\rangle \sim n^{\beta},
$$

where $\delta \tilde{\boldsymbol{r}}(n) \equiv \tilde{\boldsymbol{r}}(n)-\tilde{\boldsymbol{r}}(0)$, and $\beta \in(0,1]$ (anomalous subdiffusion). The bracket $\langle\cdots\rangle$ stands for the ensemble average over both thermal history and random environment [15]. Furthermore, the spectral dimension $d_{s}$ is related to the number of visited sites until time $n, S_{n}$, as

$$
\left\langle S_{n}\right\rangle \sim n^{d_{s} / 2},
$$

and thus $d_{s} \leq 2$. For the hypercubic lattice $\left(d_{f}=\right.$ $1,2,3, \ldots), d_{s}=1$ if $d_{f}=1$ and $d_{s}=2$ if $d_{f}=$ $2,3, \ldots$ (More precisely, a logarithmic correction appears in Eq. (2) for $d_{f}=2$. See $[12,14]$ for details).

In the DTRW on fractal lattices stated above, all the lattice points are energetically identical, while the QTM is the diffusion model on random potential landscapes. Because the QTM is the continuous time model, we denote the particle position on the fractal lattice as $\boldsymbol{r}(t) \in \mathbb{R}^{d}$. In the QTM, a particle that arrives at a site $k$ is trapped at that site for a time $\tau_{k}$ before jumping again. The trap time $\tau_{k}$ is assumed to follow a power law

$$
p(\tau) \simeq \frac{c_{0}}{\tau^{1+\mu}}, \quad \text { as } \quad \tau \rightarrow \infty, \quad(0<\mu<1)
$$

where $\mu$ is the stable index. Also, $c_{0}$ is defined by $c_{0}=c /|\Gamma(-\mu)|$, where $c$ is the scale factor and $\Gamma(-\mu)$ is the Gamma function. We assume that the trap time $\tau_{k}$ of the site $k$ is the same for each visit to this site, i.e., the random trap time $\tau_{k}$ is a quenched disorder. The origin of the power law trap time distribution [Eq. (3)] is random potential landscapes with the potential depths following 
an exponential distribution [12]. This Rapid Communication also presents numerical results for QTM on twodimensional Sierpinski gasket, for which exact values of $\beta$ and $d_{s}$ are known: $d_{s}=2 \ln 3 / \ln 5$ and $\beta=2 \ln 2 / \ln 5$ $[15]$.

On the basis of the analysis reported in [14], we approximately derive the probability density function (PDF) of the number of jumps until time $t, N_{t}$, which is an important quantity because $N_{t}$ connects the DTRW and CTRW. The following equation plays a central role:

$$
\operatorname{Prob}\left(N_{t}<n\right)=\operatorname{Prob}\left(T_{n}>t\right),
$$

where $T_{n}$ is the time when the $n$-th jump occurs and is called the $n$-th renewal time.

We start with the derivation of the PDF of $T_{n}$, then derive the PDF of $N_{t}$ through Eq. (4). Let $l_{k}^{\prime}(k=1,2, \ldots)$ denote the site index visited just after the $(k-1)$-th jump. Then, the $n$-th renewal time $T_{n}$ is expressed as

$$
T_{n}=\sum_{k=1}^{n} \tau_{l_{k}^{\prime}}
$$

Note that the same integers can appear in the sequence of site indexes $\left\{l_{k}^{\prime}\right\}_{k=1,2, \ldots, n}$, since the particle can visit the same site repeatedly. Accordingly, the trap times $\left\{\tau_{l_{k}^{\prime}}\right\}_{k=1,2, \ldots, n}$ are not mutually independent.

To handle this interdependence between trap times, we rewrite $T_{n}$ as follows [12]:

$$
T_{n}=\sum_{k=1}^{S_{n}} N_{n, k} \tau_{l_{k}}
$$

where $S_{n}$ is the number of visited sites. The indexes of these visited sites are denoted by $\left\{l_{k}\right\}_{k=1, \ldots, S_{n}}$, and $N_{n, k}$ is the number of visits to the site $l_{k}$. Note that the same integers do not appear in the sequence of site indexes $\left\{l_{k}\right\}_{k=1,2, \ldots, S_{n}}$, and thus the the trap times $\left\{\tau_{l_{k}}\right\}_{k=1,2, \ldots, S_{n}}$ are mutually independent.

Here, let us approximate $N_{n, k}$ as $N_{n, k} \approx n / S_{n}$ (i.e., we neglect fluctuations of the number of visits to each site). Moreover, in order to use the generalized central limit theorem, we rewrite Eq. (6) as

$$
T_{n} \approx \frac{n}{S_{n}} \sum_{k=1}^{S_{n}} \tau_{l_{k}}=\frac{n}{S_{n}^{1-1 / \mu}} \frac{1}{S_{n}^{1 / \mu}} \sum_{k=1}^{S_{n}} \tau_{l_{k}} \equiv a_{n} Y_{n}
$$

where $a_{n}$ and $Y_{n}$ are defined as $a_{n} \equiv n / S_{n}^{1-1 / \mu}$ and $Y_{n} \equiv$ $1 / S_{n}^{1 / \mu} \sum_{k=1}^{S_{n}} \tau_{l_{k}}$. By neglecting the fluctuations of $S_{n}$ as $S_{n} \approx\left\langle S_{n}\right\rangle$ and using Eq. (2), we further approximate $a_{n}$ as

$$
a_{n} \approx K n^{1+d_{s}(1-\mu) /(2 \mu)},
$$

where $K$ is a constant. Since $\left\{\tau_{l_{k}}\right\}_{l=1, \ldots, S_{n}}$ are mutually independent, we can use the generalized central limit theorem $[12,16]$, and find that $Y_{n}$ converges to a random variable $Y$ as $n \rightarrow \infty$ which follows the one-sided stable distribution $l_{\mu}(y)$. Thus, $T_{n}$ also follows the $l_{\mu}(y)$ after a suitable rescaling. A series expansion of $l_{\mu}(y)$ is given by $[16]$

$$
l_{\mu}(y)=\frac{-1}{\pi y} \sum_{k=1}^{\infty} \frac{\Gamma(k \mu+1)}{k !}\left(-c y^{-\mu}\right)^{k} \sin (k \pi \mu) .
$$

Next, we derive the PDF of $N_{t}$. First, let us define a rescaled variable $X_{t}$ as

$$
N_{t}=b_{t} X_{t}, \quad \text { with } \quad b_{t} \simeq\left(\frac{t}{K}\right)^{\mu / \alpha},
$$

where $\alpha \in[(1+\mu) / 2,1]$ is defined by

$$
\alpha=\mu+d_{s}(1-\mu) / 2 .
$$

This parameter $\alpha$ is important because it characterizes the deviation of the QTM from the CTRW. The PDF of $X_{t}$ is the same as that of $N_{t}$ except for the difference in the scale factor, thus we derive the PDF of $X_{t}$ instead of $N_{t}$. By using these rescaled variables $X_{t}$ and $Y_{n}$, Eq. (4) can be rewritten as

$$
\operatorname{Prob}\left(X_{t}<x\right)=\operatorname{Prob}\left(Y_{n}>x^{-\alpha / \mu}\right),
$$

where $x$ is defined by

$$
x \equiv \frac{n}{b_{t}}=\left(\frac{t}{a_{n}}\right)^{-\mu / \alpha} .
$$

Because $Y_{n}$ converges to the random variable following $l_{\mu}(y)$, the right hand side of Eq. (12) tends to an integral of $l_{\mu}(y)$ in the scaling limit $n \rightarrow \infty$ (with $x$ being fixed):

$$
\operatorname{Prob}\left(Y_{n}>x^{-\alpha / \mu}\right) \simeq \int_{x^{-\alpha / \mu}}^{\infty} l_{\mu}(y) d y
$$

Thus we obtain the PDF of $X_{t}$ by taking derivatives of Eqs. (12) and (14) with respect to $x$ :

$$
g_{\mu, \alpha}(x)=-\frac{\alpha}{\pi \mu x} \sum_{k=1}^{\infty} \frac{\Gamma(k \mu+1)}{k !}\left(-c x^{\alpha}\right)^{k} \sin (k \pi \mu) .
$$

There are two remarks. The first remark is that, since $X_{t}$ converges to a time-independent random variable that follows the $\operatorname{PDF}(15)$, we have $\left\langle N_{t}\right\rangle \sim b_{t}$. The second remark is that the above $\mathrm{PDF}$ is a one-parameter extension of the Mittag-Leffler distribution (MLD) [16, 17] for which $\alpha=1$; thus, we call it a modified MLD [14]. A qualitative difference from the MLD is that $g_{\mu, \alpha}(x)$ diverges at $x=0$ as $g_{\mu, \alpha}(x) \sim 1 / x^{1-\alpha}$. See [14] for more details. Finally, we obtain the PDF for $N_{t}$ as

$$
f_{\mu, \alpha}(n, t) \approx g_{\mu, \alpha}(x) \frac{d x}{d n}=g_{\mu, \alpha}\left(\frac{n}{b_{t}}\right) \frac{1}{b_{t}},
$$

where we used Eq. (13).

Next, we derive asymptotic formulas for the EMSD $\left\langle\delta \boldsymbol{r}^{2}(\Delta)\right\rangle$, where $\delta \boldsymbol{r}(\Delta) \equiv \boldsymbol{r}(\Delta)-\boldsymbol{r}(0)$, and the timeaveraged mean square displacement (TMSD) $\overline{\delta \boldsymbol{r}^{2}}(\Delta, t)$ 

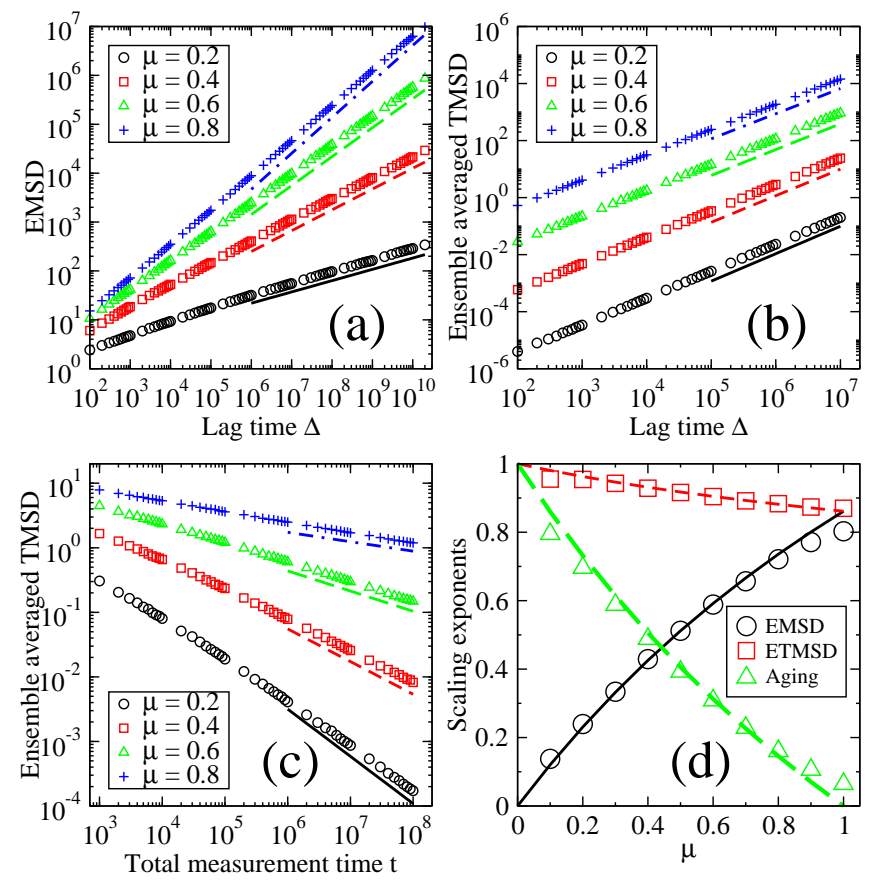

FIG. 1. (Color online) Symbols are numerical results for the QTM on the Sierpinski gasket, while curves are theoretical predictions [Eq. (17) for the EMSD and Eq. (24) for the ETMSD]. The trap time PDF is set as $p(\tau)=1 /(1+\tau / \mu)^{\mu+1}$ with $\tau \in(0, \infty)$. (a) and (b) The EMSD $\left\langle\delta \boldsymbol{r}^{2}(\Delta)\right\rangle$ and $\operatorname{ETMSD}\left\langle\overline{\delta \boldsymbol{r}^{2}}(\Delta, t)\right\rangle$ vs the lag time $\Delta$. The total measurement time $t$ is set as $t=2 \times 10^{10}$. (c) The $\operatorname{ETMSD}\left\langle\overline{\delta \boldsymbol{r}^{2}}(\Delta, t)\right\rangle$ vs the total measurement time $t$ [see Eq. (24)]. The lag time $\Delta$ is fixed as $\Delta=10^{2}$. This figure shows aging behavior of the ETMSD. (d) Scaling exponents of anomalous diffusion for the EMSD (circles and solid line) and ETMSD (squares and dashed line) vs $\mu$. The total measurement time $t$ is set as $t=2 \times 10^{13}$ for $\mu=\{0.1,0.2,0.3\}, t=2 \times 10^{11}$ for $\mu=\{0.4,0.5,0.6\}, t=2 \times 10^{9}$ for $\mu=\{0.7,0.8\}$, and $t=2 \times 10^{8}$ for $\mu=\{0.9,1.0\}$. The scaling exponent of aging for the ETMSD is also shown (triangles and long dashed line). These scaling exponents are obtained by least-square fittings (under log-log form) in the range $\Delta \in\left[10^{6}, t\right]$ for the EMSD (circles), and $\Delta \in\left[10^{5}, 10^{7}\right]$ (squares) and $t \in\left[10^{6}, 10^{8}\right]$ (triangles) for the ETMSD.

which is defined below. Here, $\Delta$ is the lag time, and $t$ is the total measurement time. The ensemble average $\langle\ldots\rangle$ is taken over both the thermal history and random environment (realizations of random fractals and the quenched disorder of traps). Using a method presented in $[7,18]$, we have

$$
\begin{aligned}
\left\langle\delta \boldsymbol{r}^{2}(\Delta)\right\rangle & \approx \int_{0}^{\infty}\left\langle\delta \tilde{\boldsymbol{r}}^{2}(n)\right\rangle f_{\mu, \alpha}(n, \Delta) d n \\
& \approx \int_{0}^{\infty}\left(x b_{\Delta}\right)^{\beta} g_{\mu, \alpha}(x) d x \sim \Delta^{\mu \beta / \alpha},
\end{aligned}
$$

where we used Eqs. (1), (10), (13) and (16). Thus, EMSD shows anomalous subdiffusion [See Fig. 1(a)]. Only when $\alpha=1$ (or, equivalently, $d_{f}=2$ ), does the scaling expo- nent of the subdiffusion coincide with that of the CTRW [7].

The TMSD, $\overline{\delta \boldsymbol{r}^{2}}(\Delta, t)$, is defined by $[3-5]$

$$
\overline{\delta \boldsymbol{r}^{2}}(\Delta, t)=\frac{1}{t-\Delta} \int_{0}^{t-\Delta} d t^{\prime}\left|\boldsymbol{r}\left(t^{\prime}+\Delta\right)-\boldsymbol{r}\left(t^{\prime}\right)\right|^{2},
$$

This TMSD is often used in single-particle tracking experiments because it is difficult in general to obtain many trajectories. We rewrite the TMSD as [19, 20]

$$
\overline{\delta \boldsymbol{r}^{2}}(\Delta, t) \approx \frac{1}{t} \sum_{k=1}^{N_{t}} H_{k}(\Delta)
$$

with

$$
H_{k}(\Delta) \equiv\left|\delta \boldsymbol{r}_{k}\right|^{2} \Delta+2 \sum_{l=1}^{k-1}\left(\delta \boldsymbol{r}_{k} \cdot \delta \boldsymbol{r}_{l}\right) \theta\left(\Delta-\left(T_{k}-T_{l}\right)\right),
$$

where $\delta \boldsymbol{r}_{k} \in \mathbb{R}^{d}$ is the displacement at time $T_{k}$, and $\theta(t)$ is defined by $\theta(t)=t$ for $t \geq 0$, otherwise $\theta(t)=0$. These equations can be derived by expressing $\boldsymbol{r}\left(t^{\prime}\right)$ as $\boldsymbol{r}\left(t^{\prime}\right)=\sum_{k=1}^{\infty} \delta \boldsymbol{r}_{k} I\left(T_{k}<t^{\prime}\right)$, where $I(\ldots)$ is the indicator function, i.e., $I(\ldots)=1$ if the inside of the bracket is satisfied, while $I(\ldots)=0$ otherwise. Then, expressing the integrand in Eq. (18), $\left|\boldsymbol{r}\left(t^{\prime}+\Delta\right)-\boldsymbol{r}\left(t^{\prime}\right)\right|^{2}$, with $\delta \boldsymbol{r}_{k}$ and the indicator function, we obtain Eq. (19).

From Eq. (19), we have

$$
\overline{\delta \boldsymbol{r}^{2}}(\Delta, t) \approx \frac{N_{t}}{t} \frac{1}{N_{t}} \sum_{k=1}^{N_{t}} H_{k}(\Delta) \rightarrow \frac{N_{t}}{t} h(\Delta)
$$

for large $t$. Here, we assume that the law of large numbers is satisfied for the summation of the random variables $H_{k}(\Delta)$. This assumption can be proved for hypercubic lattices [19] and confined systems [20], whereas a general proof seems difficult, because the correlation between displacements $\delta \boldsymbol{r}_{k}$ should be taken into account. Nevertheless, this assumption is reasonable, because it is essentially the ergodic hypothesis for the DTRW [20], and it is well accepted fact that DTRWs on fractals are ergodic [21]. The important point is that the statistical properties of the TMSD are completely determined by $N_{t}$, and we have already derived the PDF of $N_{t}$ in Eqs. (15) and (16).

The ensemble average of Eq. (21) gives

$$
\left\langle\overline{\delta \boldsymbol{r}^{2}}(\Delta, t)\right\rangle \sim t^{\frac{\mu}{\alpha}-1} h(\Delta),
$$

where we used $\left\langle N_{t}\right\rangle \sim b_{t} \sim t^{\mu / \alpha}$. On the other hand, the ensemble average of Eq. (18) gives

$$
\begin{aligned}
\left\langle\overline{\delta \boldsymbol{r}^{2}}(\Delta, t)\right\rangle & \sim \frac{1}{t} \int_{0}^{t} d t^{\prime}\left\langle\left|\boldsymbol{r}\left(t^{\prime}+\Delta\right)-\boldsymbol{r}\left(t^{\prime}\right)\right|^{2}\right\rangle \\
& \sim \frac{\Delta^{1+\mu \beta / \alpha}}{t} \int_{0}^{t / \Delta} d t^{\prime} q\left(t^{\prime}\right)
\end{aligned}
$$



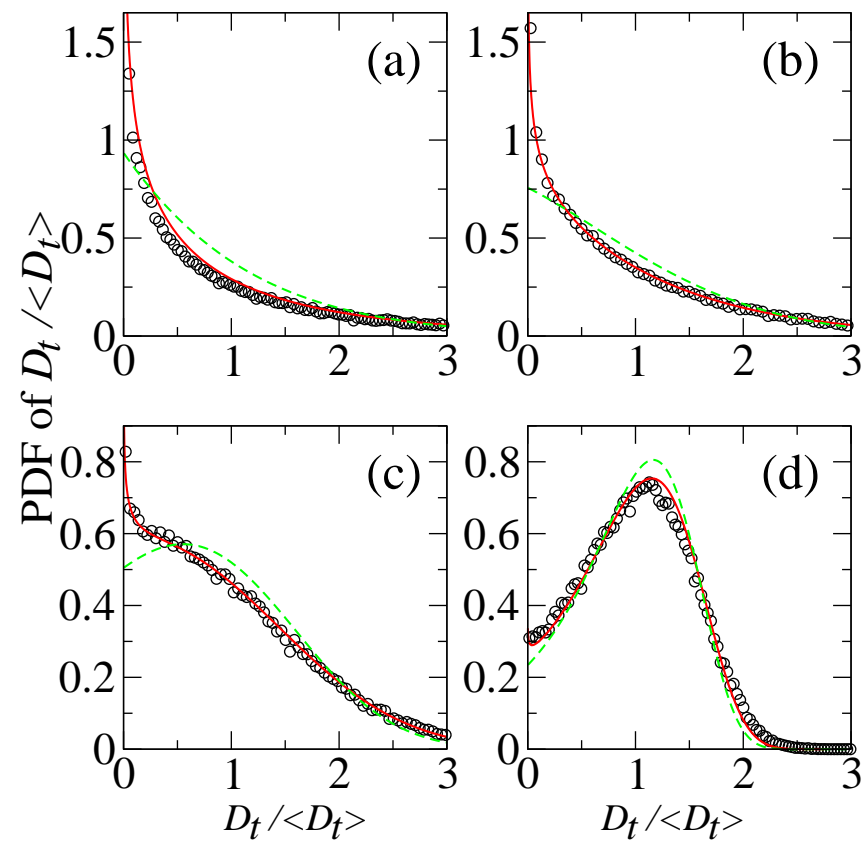

FIG. 2. (Color online) Rescaled PDF of the diffusion constant calculated simply by $D_{t} /\left\langle D_{t}\right\rangle=\overline{\delta \boldsymbol{r}^{2}}(\Delta, t) /\left\langle\overline{\delta \boldsymbol{r}^{2}}(\Delta, t)\right\rangle$ [Eq. (25)] for $\Delta=10^{5}$ and $t=2 \times 10^{9}$. The parameter $\mu$ is set as (a) $\mu=0.2$, (b) $\mu=0.4$, (c) $\mu=0.6$, and (d) $\mu=0.8$. The solid curves are the theoretical result [Eq. (15)], and the dashed curves are the MLD.

Here, we assumed a scaling hypothesis for the integrand $\left\langle\left|\boldsymbol{r}\left(\Delta\left(t^{\prime}+1\right)\right)-\boldsymbol{r}\left(\Delta t^{\prime}\right)\right|^{2}\right\rangle \sim \Delta^{\gamma} q\left(t^{\prime}\right)$ with a constant $\gamma$ and an unknown function $q\left(t^{\prime}\right)$. By setting $t^{\prime}=0$, we found $\gamma=\mu \beta / \alpha$ from Eq. (17). Note that on fractal lattices, $\left\langle\left|\boldsymbol{r}\left(\Delta\left(t^{\prime}+1\right)\right)-\boldsymbol{r}\left(\Delta t^{\prime}\right)\right|^{2}\right\rangle \neq\left\langle\boldsymbol{r}^{2}\left(\Delta\left(t^{\prime}+1\right)\right)\right\rangle-$ $\left\langle\boldsymbol{r}^{2}\left(\Delta t^{\prime}\right)\right\rangle$ in general, due to correlations between successive jump directions.

Comparing Eqs. (22) and (23), we have $\int_{0}^{t / \Delta} d t^{\prime} q\left(t^{\prime}\right) \sim$ $(t / \Delta)^{\mu / \alpha}$, and the ensemble-averaged TMSD (ETMSD) is given by

$$
\left\langle\overline{\delta \boldsymbol{r}^{2}}(\Delta, t)\right\rangle \sim \frac{\Delta^{1+(\beta-1) \mu / \alpha}}{t^{1-\mu / \alpha}}
$$

Thus, the ETMSD shows subdiffusion $\left[\Delta^{1+(\beta-1) \mu / \alpha}\right]$ as well as aging $\left(1 / t^{1-\mu / \alpha}\right)$. See also Figs. $1(\mathrm{~b})$ and (c). Note that the above formula is equivalent to that for CTRWs [7] if $\alpha=1$ (or equivalently, $d_{f}=2$ ). By contrast, if $\alpha<1$ (or equivalently, $d_{f}<2$ ), the above equation is not equivalent to that of CTRWs.

Finally, we derive the PDF of the generalized diffusion coefficient of the TMSD. From Eqs. (22) and (24), we obtain $h(\Delta)=\Delta^{1+(\beta-1) \mu / \alpha}$ and thus Eq. (21) is rewritten as

$$
\overline{\delta \boldsymbol{r}^{2}}(\Delta, t) \sim \frac{N_{t}}{t} \Delta^{1+(\beta-1) \mu / \alpha} .
$$

It follows that the generalized diffusion coefficient $D_{t}$ is given by $D_{t} \sim N_{t} / t$ and therefore $D_{t} /\left\langle D_{t}\right\rangle$ follows the same PDF as $N_{t} /\left\langle N_{t}\right\rangle$ (see Fig. 2). If the system is ergodic, this PDF converges to a delta function, that is $D_{t} \rightarrow\left\langle D_{t}\right\rangle$ as $t \rightarrow \infty$. However, this is not the case in the present model; the PDF converges to the modified MLD $g_{\mu, \alpha}(x)$ [Eq. (15)], and thus the ergodicity breaks down weakly with everlasting randomness of timeaveraged quantities $[5,7,14,17,19,20,22-24]$.

In summary, the QTM on fractal lattices was investigated and anomalous subdiffusion was found for both EMSD and TMSD. It is also shown that this system shows weak ergodicity breaking, and the diffusion constant of the TMSD becomes a random variable following the modified MLD. This modified MLD has a divergent peak at the origin, which means that there are trajectories with small diffusivity much more frequently in the QTM than in the CTRW.

We also show that if the spectral dimension $d_{s}$ of the fractal lattice satisfies $d_{s}<2$, the QTM cannot be reduced to the CTRW; in other words, the CTRW is physically irrelevant as a model of a random walk on random potential energy landscapes and we have to use the QTM instead of the CTRW (though, if $d_{s}$ is close to 2 , the CTRW is a good approximation of the QTM). Only if $d_{s}=2$ the QTM is asymptotically equivalent to the CTRW. Finally, it is worth mentioning that, even though we focused on the TMSD as a time-averaged observable, the weak ergodicity breaking and the modified MLD must appear for a wide class of observables [19, 20].
[1] E. Barkai, Y. Garini, and R. Metzler, Physics Today 65 (8), 29 (2012); F. Höfling and T. Franosch, Rep. Prog. Phys. 76, 046602 (2013).

[2] R. Metzler, J.-H. Jeon, A. G. Cherstvy, and E. Barkai, Phys. Chem. Chem. Phys. 16, 24128 (2014).

[3] I. Golding and E. C. Cox, Phys. Rev. Lett. 96, 098102 (2006); J.-H. Jeon, V. Tejedor, S. Burov, E. Barkai, C. Selhuber-Unkel, K. Berg-Sørensen, L. Oddershede, and R. Metzler, ibid. 106, 048103 (2011); S. Burov, J. Jeon, R. Metzler, and E. Barkai, Phys. Chem. Chem. Phys. 13, 1800 (2011).

[4] T. Akimoto, E. Yamamoto, K. Yasuoka, Y. Hirano, and
M. Yasui, Phys. Rev. Lett. 107, 178103 (2011); T. Uneyama, T. Akimoto, and T. Miyaguchi, J. Chem. Phys. 137, 114903 (2012); J.-H. Jeon, H. M.-S. Monne, M. Javanainen, and R. Metzler, Phys. Rev. Lett. 109, 188103 (2012)

[5] Y. He, S. Burov, R. Metzler, and E. Barkai, Phys. Rev. Lett. 101, 058101 (2008); A. Lubelski, I. M. Sokolov, and J. Klafter, ibid. 100, 250602 (2008); T. Neusius, I. M. Sokolov, and J. C. Smith, Phys. Rev. E 80, 011109 (2009); P. Massignan, C. Manzo, J. A. Torreno-Pina, M. F. García-Parajo, M. Lewenstein, and G. J. Lapeyre, Phys. Rev. Lett. 112, 150603 (2014); F. Thiel, F. Flegel, 
and I. M. Sokolov, ibid. 111, 010601 (2013); F. Thiel and I. M. Sokolov, Phys. Rev. E 89, 012136 (2014); J. H. P. Schulz, E. Barkai, and R. Metzler, Phys. Rev. X 4, 011028 (2014).

[6] A. V. Weigel, B. Simon, M. M. Tamkun, and D. Krapf, Proc. Natl. Acad. Sci. U.S.A 108, 6438 (2011).

[7] Y. Meroz, I. M. Sokolov, and J. Klafter, Phys. Rev. E 81, 010101 (2010).

[8] S. M. A. Tabei, S. Burov, H. Y. Kim, A. Kuznetsov, T. Huynh, J. Jureller, L. H. Philipson, A. R. Dinner, and N. F. Scherer, Proc. Natl. Acad. Sci. U.S.A 110, 4911 (2013).

[9] E. Yamamoto, T. Akimoto, M. Yasui, and K. Yasuoka, Sci. Rep. 4, 4720 (2014).

[10] A. Bancaud, S. Huet, N. Daigle, J. Mozziconacci, J. Beaudouin, and J. Ellenberg, EMBO J. 28, 3785 (2009).

[11] J. Machta, J. Phys. A 18, L531 (1985).

[12] J. Bouchaud and A. Georges, Phys. Rep. 195, 127 (1990).

[13] S. Burov and E. Barkai, Phys. Rev. Lett. 98, 250601 (2007); Phys. Rev. E 86, 041137 (2012).

[14] T. Miyaguchi and T. Akimoto, Phys. Rev. E 83, 031926 (2011).

[15] S. Havlin and D. Ben-Avraham, Adv. Phys. 51, 187 (2002).

[16] W. Feller, An Introduction to Probability Theory and its Applications, 2nd ed., Vol. II (Wiley, New York, 1971).

[17] J. Aaronson, An Introduction to Infinite Ergodic Theory (American Mathematical Society, Province, 1997); T. Akimoto and T. Miyaguchi, Phys. Rev. E 82, 030102 (2010).
[18] G. Zumofen, J. Klafter, and A. Blumen, The Journal of Chemical Physics 79, 5131 (1983); A. Blumen, J. Klafter, B. S. White, and G. Zumofen, Phys. Rev. Lett. 53, 1301 (1984).

[19] T. Miyaguchi and T. Akimoto, Phys. Rev. E 83, 062101 (2011).

[20] T. Miyaguchi and T. Akimoto, Phys. Rev. E 87, 032130 (2013).

[21] J. Szymanski and M. Weiss, Phys. Rev. Lett. 103, 038102 (2009).

[22] A. Rebenshtok and E. Barkai, Phys. Rev. Lett. 99, 210601 (2007); J.-H. Jeon, E. Barkai, and R. Metzler, J. Chem. Phys. 139, 121916 (2013); D. Froemberg and E. Barkai, Phys. Rev. E 87, 030104 (2013); Eur. Phys. J. B 86, 331 (2013); A. G. Cherstvy, A. V. Chechkin, and R. Metzler, New Journal of Physics 15, 083039 (2013).

[23] T. Akimoto and T. Miyaguchi, Phys. Rev. E 87, 062134 (2013); J. Stat. Phys. 157, 515 (2014).

[24] The weak ergodicity breaking is usually defined through a mismatch of time-averaged and ensemble-averaged quantities without any division of the phase space into mutually inaccessible regions $[2,25]$. In addition, the systems such as the QTM and CTRW show the everlasting randomness of time-averaged quantities as stated in the main text. In contrast, there are systems in which ergodicity breaks down weakly without such randomness of time-averaged quantities. See, for example, [26].

[25] J. P. Bouchaud, J. Phys. I France 2, 1705 (1992).

[26] A. Fuliński, Phys. Rev. E 83, 061140 (2011); F. Thiel and I. M. Sokolov, ibid. 89, 012115 (2014); J.-H. Jeon, A. V. Chechkin, and R. Metzler, Phys. Chem. Chem. Phys. 16, 15811 (2014). 